GLOBAL SCIENCE AND TECHNOLOGY (ISSN 1984 - 3801)

\title{
USO DE GEOESTATÍSTICA NO ESTUDO DA VARIABILIDADE ESPACIAL DA CAPACIDADE DE TROCA DE CÁTIONS DO SOLO
}

\author{
Rubson Natal Ribeiro Sibaldelli ${ }^{1 *}$, Josirley de Fátima Corrêa Carvalho ${ }^{2}$, Maria Cristina Neves de \\ Oliveira $^{3}$
}

\begin{abstract}
RESUMO: A Capacidade de Troca de Cátions (CTC) é um importante atributo dos solos e caracteriza a quantidade de cátions que um solo é capaz de reter por unidade de peso ou volume e influencia a disponibilidade de nutrientes às plantas. O presente trabalho teve como objetivo avaliar a estrutura da dependência espacial da CTC Total. O levantamento amostral foi realizado no município de Campos dos Goytacazes, no estado do Rio de Janeiro. Foram coletadas amostras de forma regionalizada, com trado holandês, em uma malha regular, distando 900 metros para cada coordenada geográfica: norte e leste. Esta malha foi idealizada com espaçamento de 50 metros, totalizando 273 amostras que, com a retirada dos dados discrepantes após as análises iniciais, foram reduzidas para 240 amostras. Os dados foram submetidos às análises descritivas exploratórias, com o uso do box-plot, testes de normalidade e gráficos de controle. Posteriormente, foram realizadas as análises geoestatísticas, retirando-se as tendências linear e quadrática, determinação do semivariograma que melhor representava os dados, krigagem ordinária e construção de mapas da área amostral. Observou-se que a CTC possui dependência espacial, que o semivariograma que melhor representou os dados foi o modelo exponencial o que possibilitará a redução no número de amostras de solo em trabalhos futuros.
\end{abstract}

Palavras-chave: amostras georreferenciadas, CTC, dependência espacial, krigagem ordinária, variabilidade.

\section{GEOSTATISTICS USE IN THE STUDY OF SPATIAL VARIABILITY OF SOIL CATION EXCHANGE CAPACITY.}

ABSTRACT: Cation Exchange Capacity (CEC) is an important soil attribute and represents the quantity of cations that a soil is capable of holding per unit of weight or volume and influences the availability of nutrients to plants. This study aimed to evaluate the structure of the spatial dependence of CEC. The sample survey was conducted in the municipality of Campos dos Goytacazes in the state of Rio de Janeiro, samples were collected in a regionalized way, using a Dutch auger in a regular grid, lying 900 meters for each geographic coordinate: North and East. This mesh was idealized with spacing of 50 meters, totaling 273 samples that, after the initial analyzes, were reduced to 240 samples by the removal of outliers. The data were submitted to descriptive exploratory analyzes, using the boxplot, normality tests and control charts. Subsequently, geostatistical analyzes were performed by withdrawing the linear and quadratic trends, determination of the semivariogram that best represented the data, ordinary kriging and building of maps of the sampled area. CTC had spatial dependence, exponential model was the best semivariogram to represent the data wich will enable reduction of the number of soil samples in future studies.

Key-words: spatial dependence, georeferenced sample, ordinary kriging, variability, CEC.

\footnotetext{
${ }_{1}^{1}$ Mestrando no Programa de Pós-graduação em Engenharia Ambiental. Universidade Tecnológica Federal do Paraná - UTFPR. *E-mail: rubson.sibaldelli@embrapa.br. Autor para correspondência.

${ }^{2}$ Pós-doutoranda/ CNPq/Embrapa Soja, Londrina - PR.

${ }^{3}$ Pesquisadora - Embrapa Soja, Londrina - PR.
}

Recebido em: 15/04/2014. Aprovado em: 01/10/2014.

Gl. Sci Technol, Rio Verde, v.08, n.01, p.141 - 156, jan/abr. 2015. 


\section{INTRODUÇÃO}

A aplicação de tecnologia associada à variabilidade espacial e temporal faz-se necessária e, sobretudo, na pesquisa agrícola, que estuda o solo e a sua capacidade produtiva. $\mathrm{O}$ conhecimento da variabilidade das propriedades do solo e das culturas no espaço e no tempo é considerado, atualmente, o princípio básico para o manejo preciso das áreas agrícolas, qualquer que seja sua escala. Para análise dos atributos dos solos, consideram-se áreas relativamente pequenas, as quais são amostradas aleatoriamente. Contudo, ao considerar essas pequenas áreas como uniformes quanto aos seus atributos, podem-se interpretar erroneamente as respostas obtidas às questões existentes, pois a hipótese de ocorrência de dependência espacial estará sendo ignorada (GREGO; VIEIRA 2005).

A preocupação com a variabilidade experimental do ponto de vista estatístico vem de longa data, com os experimentos de campo, sendo dispostos em blocos ao acaso e os tratamentos aleatorizados e repetidos na área experimental. Esta metodologia foi questionada por alguns autores e recomendada por outros com algumas modificações, pois não se obtinham resultados satisfatórios dos efeitos dos tratamentos estudados, devido à variabilidade experimental e à ausência da posição geográfica das amostras (VIEIRA et al., 1981).

Metodologias para análise dos dados que apresentam aspectos aleatórios e espaciais, e que avaliam a dependência espacial são abordadas pela geoestatística. A geoestatística compreende o conjunto de métodos que tem, por objetivo, a estimativa de valores de um atributo, que são correlacionados no tempo e/ou espaço (KOPPE et al., 2006). A análise geoestatística permite detectar a existência da variabilidade e distribuição espacial das medidas estudadas e constitui-se numa importante ferramenta na análise e descrição detalhada da variabilidade das propriedades do solo (VIEIRA, 2000;
CARVALHO; SILVEIRA; VIEIRA, 2002; VIEIRA et al., 2002). Com base nas coordenadas geográficas do ponto amostrado, pode-se analisar os dados, possibilitando, representar a área com maior detalhamento.

O nível de detalhamento é obtido por meio da distância entre pontos de amostragem e depende tanto da propriedade a ser analisada quanto da escala de trabalho (tamanho da área amostrada), sendo a definição do tamanho da grade amostral uma característica importante da geoestatística. No Brasil, até a década de 70, os atributos físicos e químicos dos solos eram avaliados em delineamentos experimentais como dados independentes. Após anos de uso deste método, descobriu-se uma dependência espaço-temporal natural nos solos, fazendo com que ferramentas que incluem a posição geográfica das amostras como a geoestatística tornassem-se essenciais. Oliveira et al. (2009), Souza et al. (2010a) e Santos, Contijo e Nicole (2012), afirmaram que atributos químicos do solo apresentam dependência espacial, já Souza et al. (2010b), Carvalho et al. (2011) e Cavalcante et al. (2011) encontraram dependência espacial em atributos físicos de solos.

Neste trabalho, foi avaliada a Capacidade de Troca de Cátions (CTC), definida como a quantidade de cátions que um solo é capaz de reter por unidade de peso ou volume. Para fins de química e classificação de solos, os resultados são expressos por peso de solo. Em fertilidade, são comuns ambas as maneiras, devendo-se dar preferência à expressão por volume. Ainda pode ser definida como sendo a soma total de cátions que um solo pode reter na superfície coloidal prontamente disponível à assimilação pelas plantas. Baseado no exposto acima, o presente estudo objetivou avaliar a estrutura da dependência espacial da CTC Total.

\section{MATERIAL E MÉTODOS}

Os dados de CTC Total - $\mathrm{cmol}_{\mathrm{c}} \mathrm{dm}^{-3}$ (Cálcio, Magnésio, Potássio, Hidrogênio e 
Alumínio) usados no presente trabalho foram obtidos de amostras coletadas na camada de 0-20 cm pela Embrapa Solos, na Fazenda Angra (Estação Experimental de Campos de Goytacazes) pertencente a PESAGRO/RIO e analisadas no laboratório da Embrapa Solos seguindo a metodologia proposta em Embrapa (1997). Os solos desta fazenda cultivados por mais de 100 anos com a cultura da cana-de-açúcar, possuem predominância da classe dos Cambissolos Háplicos abrangendo 46,6\% da área, com suas características morfológicas, físicas e químicas encontradas em Embrapa (2003) e Oliveira (2003). A área amostrada encontrase entre as coordenadas geográficas

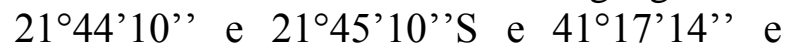
$41^{\circ} 17^{\prime} 50^{\prime}$ 'O (Figura 1).
A amostragem do solo foi realizada com trado holandês, em uma malha regular espaçada em 50 metros, distando 900 metros para cada coordenada geográfica: norte e leste, totalizando 273 amostras.

Inicialmente, os dados foram submetidos à análise exploratória descritiva e gráfica por meio de mapas da amostragem, testes de aderência à normalidade, gráficos de box-plot, cartas de controle, histogramas e testes de correlação espacial. Posteriormente, os dados foram analisados, usando-se ferramentas geoestatísticas como semivariograma, método de interpolação pela krigagem ordinária, validação por JackKnifing (VIEIRA; CARVALHO; GONZÁLES, 2010), e mapas de isolinhas.

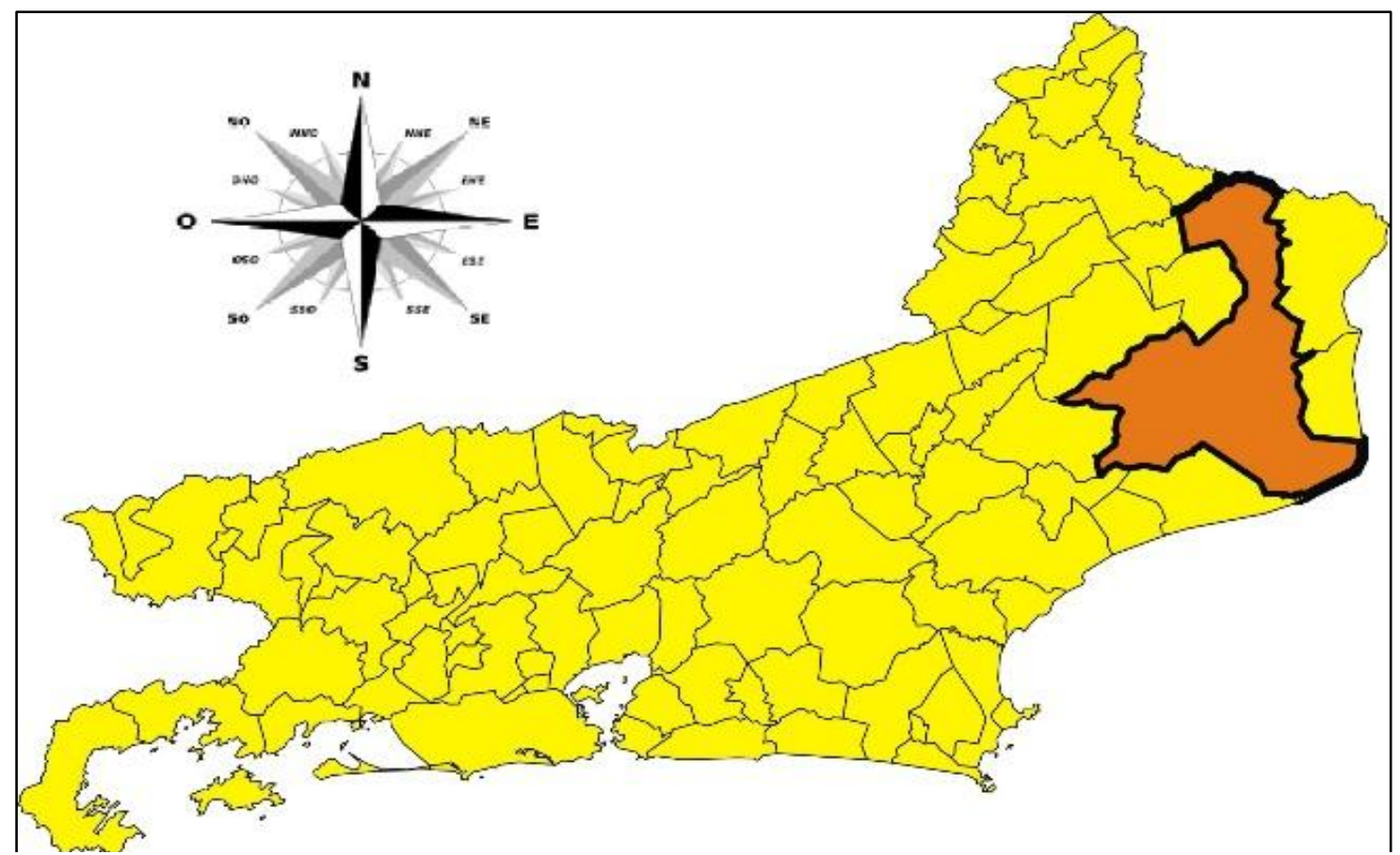

Figura 1. Mapa do Estado do Rio de Janeiro, com o município de Campos dos Goytacazes em destaque.

foram utilizados os programas computacionais: Statsoft, inc. (2004), Statistica (Data Analysis Software System), versão 7. www.statsoft.com; Surfer versão 9.9.785 - mar 26.2010, surface mapping system, www.goldensoftware.com; GEOEST - Software para cálculos de geoestatística, Vieira (2000), IAC, Campinas.

\section{RESULTADOS E DISCUSSÃO}

Anterior a qualquer método estatístico ou geoestatístico é essencial que se conheça a distribuição espacial, a presença de valores anômalos e a variabilidade dos dados por meio de estatísticas descritivas. Considerando-se a CTC Total usada no presente estudo, pôde-se observar que os valores para média e mediana dos diferentes 
conjuntos de dados (com e sem outliers) curtose aproximando-se de zero (Tabela 1). foram próximos, com leve assimetria e

Tabela 1. Estatísticas descritivas para CTC Total $\left(\mathrm{cmol}_{\mathrm{c}} \mathrm{dm}^{-3}\right), 0-20 \mathrm{~cm}$ do solo com e sem outliers.

\begin{tabular}{lcccc}
\hline Estatísticas & Com Outliers & Com Outliers & Com Outliers & Sem Outliers \\
\hline $\mathrm{N}^{\circ}$ Observações & 273 & 254 & 250 & 240 \\
Média & 12,86 & 12,98 & 13,02 & 13,10 \\
Mediana & 12,57 & 13,06 & 13,07 & 13,08 \\
Mínimo & 5,55 & 7,88 & 8,24 & 8,72 \\
Máximo & 21,30 & 17,82 & 17,64 & 17,32 \\
Amplitude Total & 15,75 & 9,94 & 9,40 & 8,60 \\
Variância & 6,64 & 4,19 & 3,85 & 3,14 \\
Coeficiente de Assimetria & $-0,24$ & $-0,14$ & $-0,09$ & 0,06 \\
Coeficiente de Curtose & 0,81 & 0,10 & 0,03 & $-0,13$ \\
Desvio Padrão & 2,58 & 2,05 & 1,96 & 1,77 \\
Coeficiente de Variação $(\%)$ & 20,06 & 15,79 & 15,05 & 13,51 \\
\hline
\end{tabular}

É conhecido que a presença de dados discrepantes pode mascarar os resultados de pesquisa e vários são os métodos estatísticos que auxiliam na tomada de decisão com relação à escolha do conjunto de dados capazes de gerar estimativas confiáveis. Dentre tais métodos, incluem-se testes de normalidade, gráficos tridimensionais, gráficos de dispersão nos eixos x e y, gráficos box-plot e cartas de controle. No presente estudo, foram utilizados gráficos de box-plot e as cartas de controle para se detectar presença ou ausência de outliers.

Além disso, foram aplicados os testes de normalidade de KolmogorovSmirnov e Shapiro e Wilk. Outliers foram identificados e retirados do conjunto de dados gradativamente por meio de análises de boxplot, obtendo-se um conjunto de dados com 250 amostras. Os dados retirados foram na sua maioria provenientes das áreas nas quais ocorreram alagamentos das lagoas ao sul da área pesquisada e ao norte pelo Rio Paraíba do Sul na época das enchentes (OLIVEIRA, 2003) e, os valores retirados na direção norte estão relacionados ao local onde foi depositado o calcário ali distribuído (Figuras 2A a D).

Embora no box-plot com 250 amostras (Figura 3) a presença de outliers não fosse mais observada, os testes de normalidade de Shapiro e Wilk (Tabela 2) e as cartas de controle indicaram ausência de normalidade, devido à presença de outliers. Desta forma, considerou-se este agrupamento ainda com presença de outliers. Promoveu-se então a retirada de mais dez pontos, originando um novo conjunto de dados de 240 pontos (Figuras 2D e 3), este sim considerado sem outliers. 

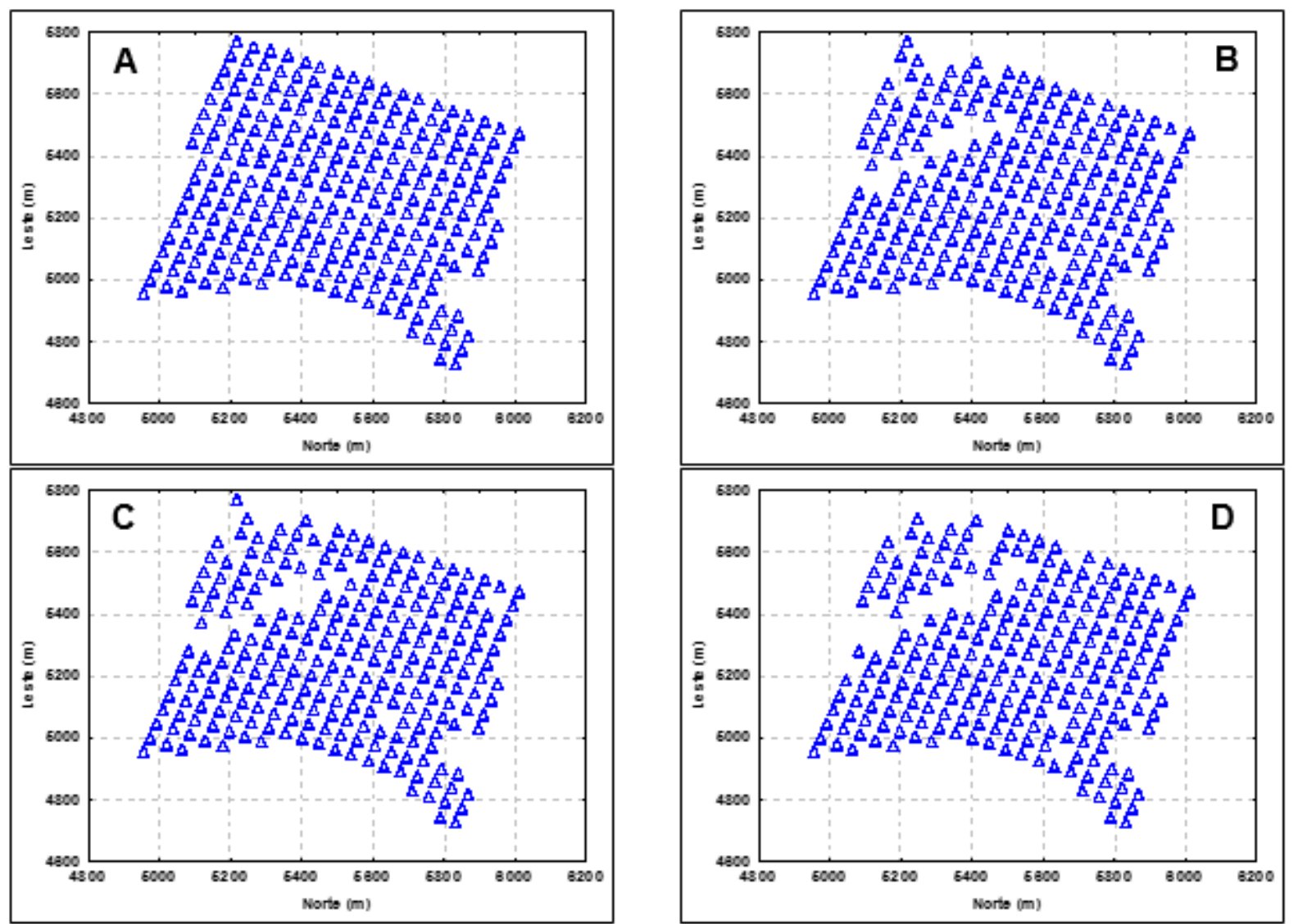

Figura 2. Mapa de amostragem em malha regular, com 273 amostras (A), 254 e 250 amostras (B, C), na presença de outliers, e 240 amostras (D) sem a presença de outliers para CTC Total $\left(\mathrm{cmol}_{\mathrm{c}} \mathrm{dm}^{-3}\right)$, na camada de $0-20 \mathrm{~cm}$ do solo.

Tabela 2. Resultados dos testes de aderência a curva normal para CTC Total $\left(\mathrm{cmol}_{\mathrm{c}} \mathrm{dm}^{-3}\right)$, 0$20 \mathrm{~cm}$ do solo com e sem outliers.

\begin{tabular}{ccccccccc}
\hline \multirow{2}{*}{ Mét } & \multicolumn{2}{c}{ Com Outliers } & \multicolumn{2}{c}{ Com Outlier } & \multicolumn{2}{c}{ Com Outliers } & \multicolumn{2}{c}{$\begin{array}{c}\text { Sem Outliers } \\
273 \text { amostras }\end{array}$} \\
\cline { 2 - 9 } & \multicolumn{2}{c}{ 254 amostras } & \multicolumn{2}{c}{250 amostras } & \multicolumn{2}{c}{240 amostras } \\
\hline \multirow{2}{*}{ KS } & $\mathrm{d}=0,070$ & $\mathrm{p}<0,150$ & $\mathrm{~d}=0,055$ & $\mathrm{p}>.20$ & $\mathrm{~d}=0,05$ & $\mathrm{p}>.20$ & $\mathrm{~d}=0,05$ & n.s. \\
$\mathrm{SW}$ & $\mathrm{W}=0,98$ & $\mathrm{p}=0,0004$ & $\mathrm{~W}=0,987$ & $\mathrm{p}=0,02$ & $\mathrm{~W}=0,99$ & $\mathrm{p}=0,04$ & $\mathrm{~W}=0,99$ & $\mathrm{p}=0,187$ \\
\hline
\end{tabular}

Mét=Métodos; KS= Kolmogorov-Smirnov; $\mathrm{SW}=$ Shapiro e Wilk; VT= Valor do Teste; Prob= Probabilidade.

Depois da retirada dos outliers houve redução na variância e no coeficiente de variação. Alguns autores afirmam que, embora a normalidade dos dados não seja exigência do método geoestatístico, os coeficientes de assimetria e curtose devem se aproximar de zero e os semivariogramas devem ter patamares bem definidos (HOAGLIN; MOSTELLER; TUKEY, 1992; CARVALHO; VIEIRA, 2001). 


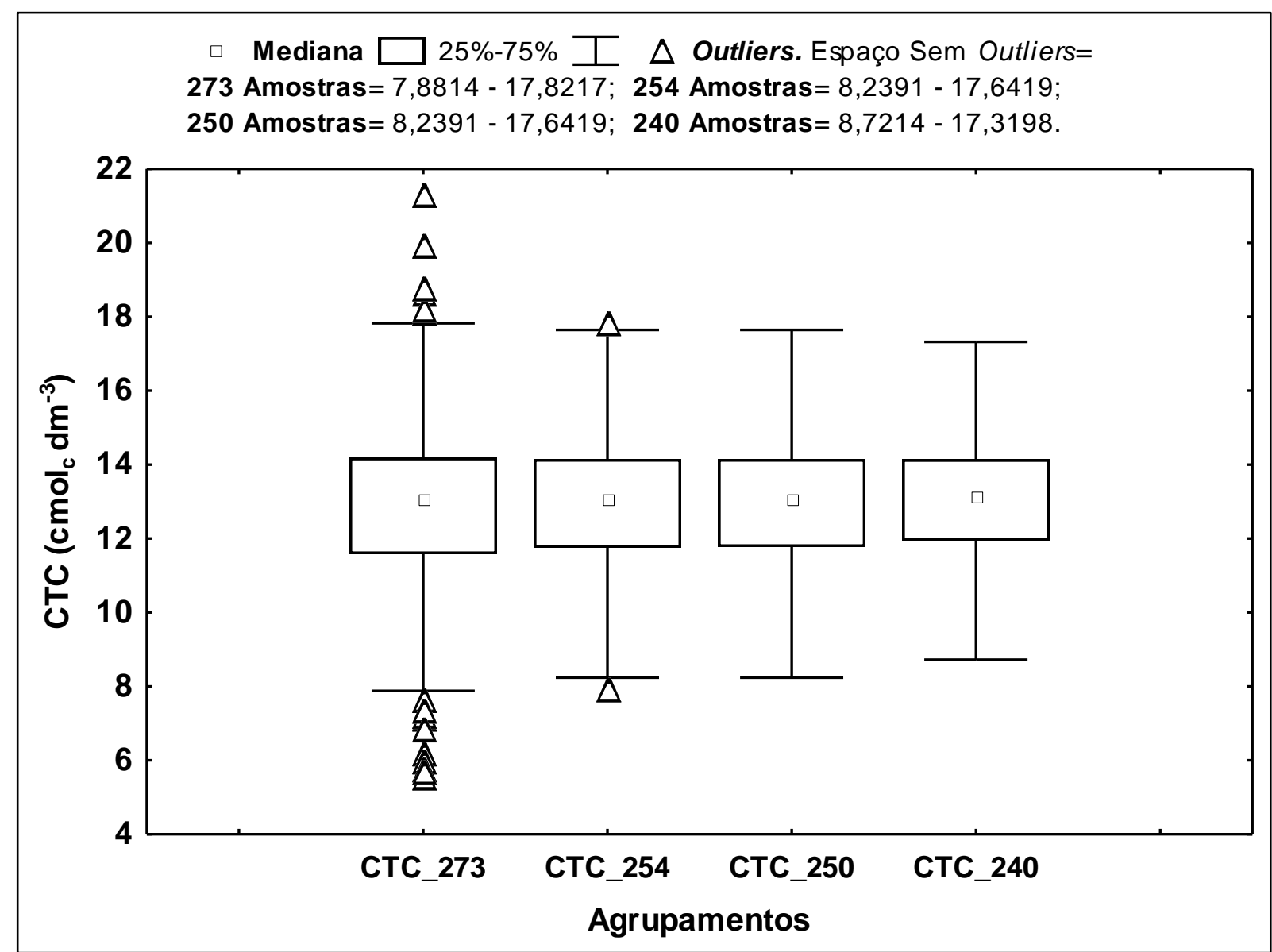

Figura 3. Box-plot para CTC Total $\left(\mathrm{cmol}_{\mathrm{c}} \mathrm{dm}^{-3}\right)$, com 273, 254, 250 e 240 amostras.

As cartas de controle (Figura 4) foram realizadas com o maior grau de rigor no intervalo de $(-3 \sigma ;+3 \sigma)$ como limites inferior e superior, em que $\sigma$ é o valor do desvio padrão populacional. Pontos encontrados dentro da faixa, acima e abaixo do valor médio, indicam ausência de outliers somente no agrupamento com 240 amostras (Figura 4D), sendo, portanto, este o conjunto de dados escolhido para as análises geoestatísticas, apresentando ainda valores de assimetria menores e curtose mais suave que os demais conjuntos (Tabela 1), conforme indicado pela distribuição de frequências sob a curva normal (Figura 5). Este conjunto de dados com 240 amostras mostra-se de acordo com a quantia mínima de pontos da amostragem (100 pontos), conforme Souza et al. (2014).

Análises para verificação da correlação espacial entre a CTC e as direções norte e leste indicaram baixa tendência linear negativa e positiva, respectivamente, em todos os conjuntos de dados (Figura 6). Os valores da correlação linear [273amostras $=$ 0,1181 (norte); 240 amostras $=-0,2972$ (norte); 273 amostras $=0,0646$ (leste); 240 amostras $=0,1087$ (leste)] indicam que ao serem retirados os outliers do conjunto de dados, a inclinação da reta aumenta.

A correlação existente nos conjuntos com 273 e 254 amostras (Figura 6A a D) possui correlação negativa na direção Norte (Figura 6A) e positiva na direção Leste (Figura 6C), sendo que ela se mostra presente porém fraca, pois no caso do conjunto de 273 amostras e direção Norte, os valores decrescem à proporção de $1,24 \%$ a cada 200 metros que se caminha nesta direção. Já na direção Leste, o mesmo conjunto de dados tem valores que aumentam na proporção de $1,54 \%$ a cada 200 metros. 

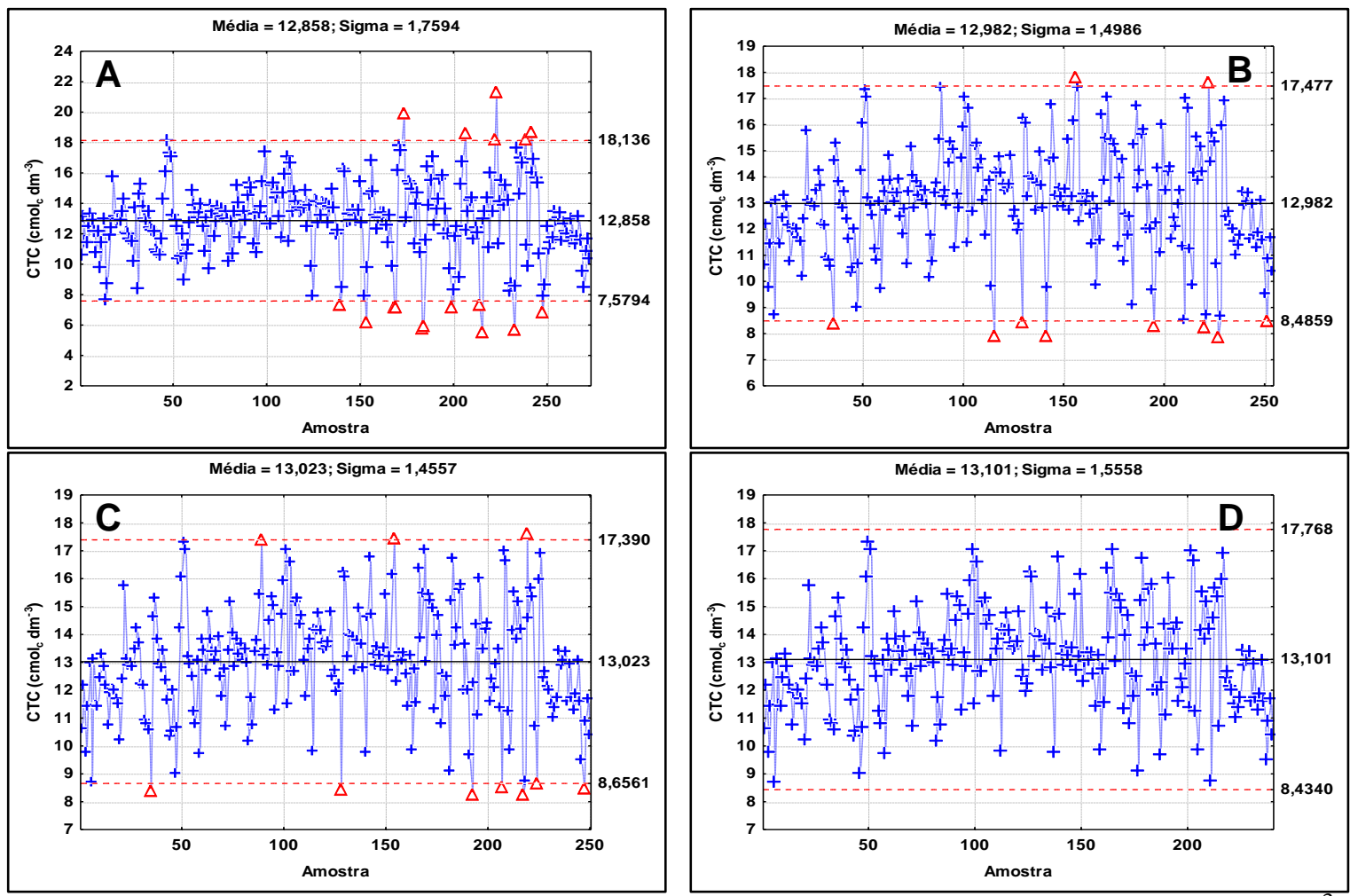

Figura 4. Cartas de controle, no intervalo de $(-3 \sigma ;+3 \sigma)$ para CTC Total $\left(\mathrm{cmol}_{\mathrm{c}} \mathrm{dm}^{-3}\right)$, na camada de 0-20 cm do solo, com 273 (A), 254 (B) e 250 amostras (C) na presença de outliers e 240 amostras (D) sem outliers.
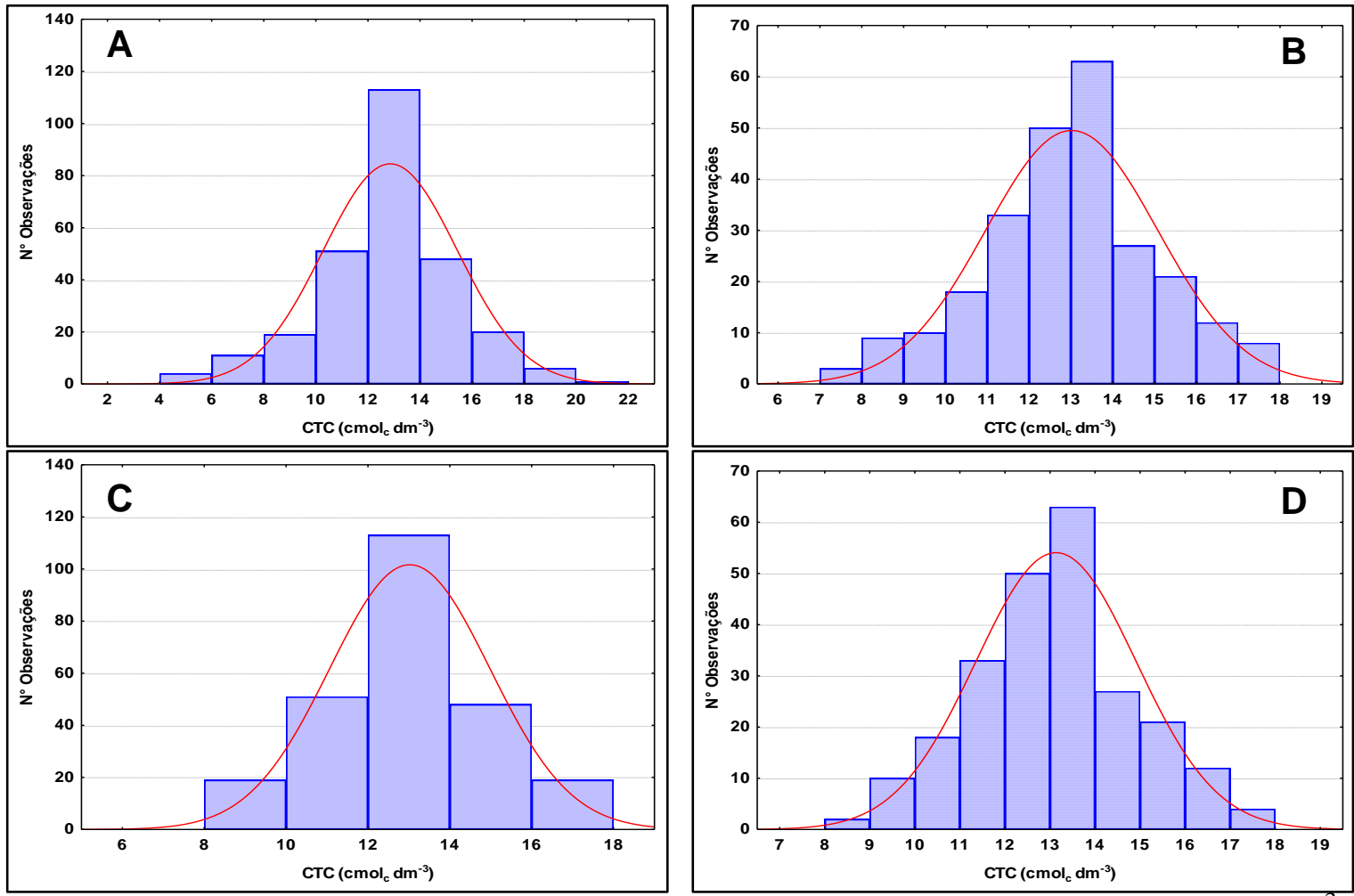

Figura 5. Distribuição de frequência sob a curva normal para CTC Total $\left(\mathrm{cmol}_{\mathrm{c}} \mathrm{dm}^{-3}\right)$, na camada 0-20 cm do solo, com outliers em 273 (A), 254 (B) e 250 amostras (C) e sem outliers em 240 amostras (D).

No conjunto de dados com 254 amostras (Figuras 6B e D), as proporções de 
decréscimo em direção ao Norte e acréscimo respectivamente, em 200 metros percorridos. em direção ao Leste são $1,54 \%$ e 1,23\%,
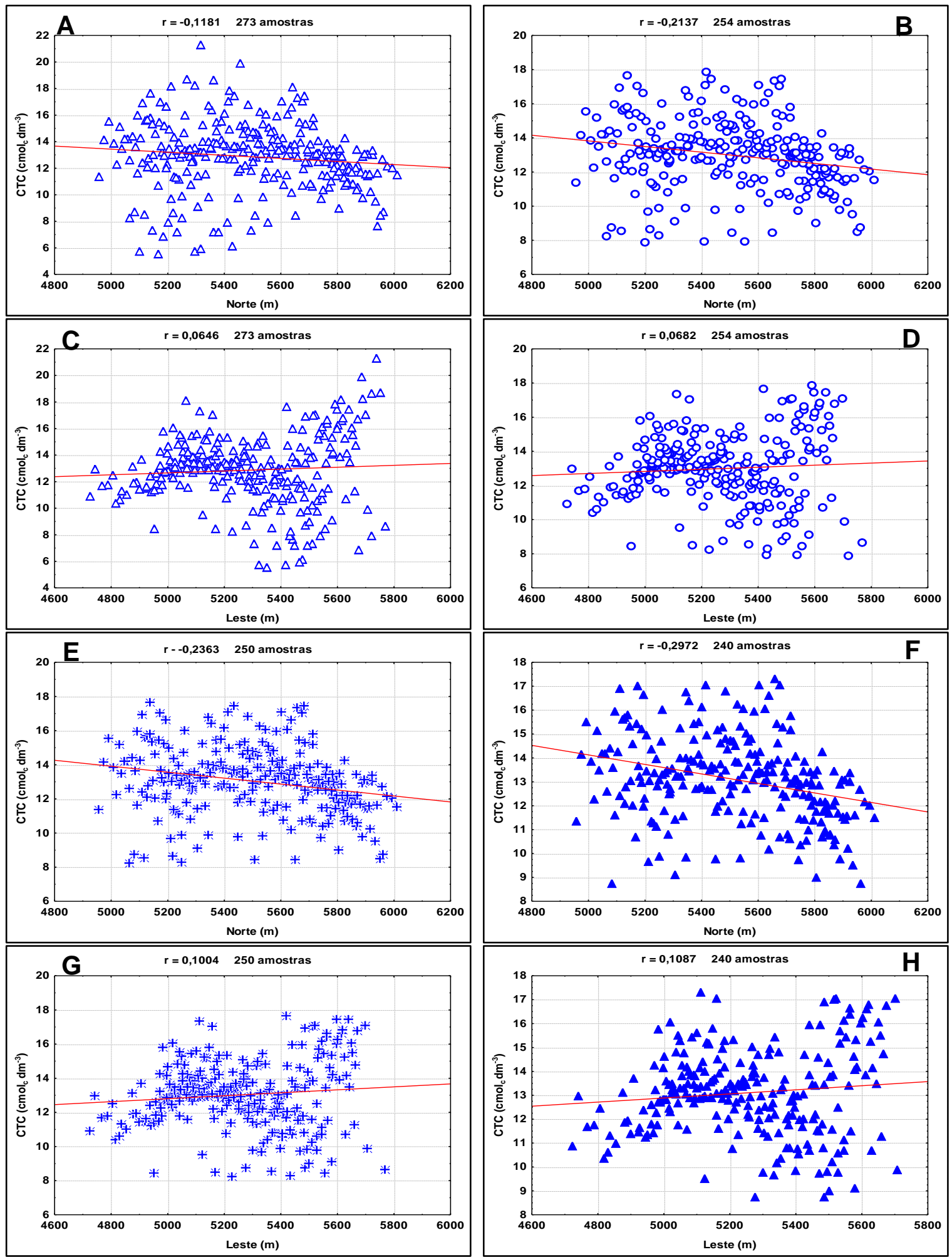

Figura 6. Correlação para as posições geográficas Norte (A, B) e Leste (C, D), com 273 (A, C) e 254 (B, D) amostras. Posições geográficas Norte (E, F) e Leste (G, H), com 250 (E, G) e $240(\mathrm{~F}, \mathrm{H})$ amostras. CTC Total $\left(\mathrm{cmol}_{\mathrm{c}} \mathrm{dm}^{-3}\right)$, na camada de $0-20 \mathrm{~cm}$ do solo. 
Os conjuntos com 250 e 240 amostras (Figura 6E a H) possuem correlação negativa na direção Norte e positiva na direção Leste, assim como os conjuntos avaliados anteriormente (Figura 6A a D). A correlação se apresenta fraca no conjunto com 250 amostras e direção Norte (Figura $6 \mathrm{E})$, cujos valores decrescem a proporção de $1,5 \%$ a cada 200 metros que se caminha nesta direção. Na direção Leste (Figura 6G), o mesmo conjunto de dados tem valores que aumentam na proporção de $2,13 \%$ a cada 200 metros. No conjunto de dados com 240 amostras (Figuras 6F e $\mathrm{H}$ ), as proporções de decréscimo em direção ao Norte e acréscimo em direção ao Leste são $1,66 \%$ e 2,09\%, respectivamente, em 200 metros percorridos em cada direção.

Para verificar a existência de um padrão espacial para a CTC Total, utilizaramse as estimativas dos parâmetros dos semivariogramas exponencial, gaussiano e esférico, como o efeito pepita, o patamar e o alcance. Após a determinação da semivariância, realizou-se a definição do tamanho do passo a ser dado (distância teórica entre amostras). Foram escolhidos os passos com 40, 56 e 80 metros.
Observou-se que os dados apresentam tendência e não um patamar estável (Figura 7). Portanto, houve a necessidade da retirada de tal tendência, em ambas dimensões para passos (40, 56 e 80 metros) pela análise de regressão linear e quadrática. Estabeleceu-se o passo de 56 metros (visto a maior regularidade gráfica desta dimensão de passo), retirando-se as tendências linear e quadrática, cujos coeficientes de determinação do ajuste foram 9,84\% e $15,46 \%$ respectivamente (Tabela 3). A escolha da retirada de tendência quadrática foi realizada assumindo nesta escolha $o$ critério do maior coeficiente de determinação.

No conjunto com 240 amostras, realizou-se o ajuste dos três modelos de semivariogramas aos dados da CTC Total (Tabela 4), apresentando dependência espacial bem definida. O menor valor de efeito pepita $(0,89)$, o maior índice de dependência espacial (IDE-68\%) e o maior patamar foram obtidos com o modelo exponencial, concordando com Silva Junior et al. (2013) que trabalharam com atributos do solo. O IDE é dado por Cambardella et al. (1994) como sendo: (Eq. 1)
As opiniões na literatura são divergentes com relação à seleção de modelos de semivariogramas, existindo aqueles que preconizam que o melhor modelo é aquele que tem menor efeito pepita e outros que selecionam modelos com variância mínima, resíduos nulos e correlação próxima de $r=1$ (VIEIRA et al., 1983), entre outras estatísticas obtidas pelo método de JackKnifing (VIEIRA, 2000). Bolfe, Gomes e Fontes (2007) enfatizam que quanto maior a diferença entre o efeito pepita e o patamar, maior a continuidade do fenômeno e menor a variância da estimativa, ou seja, maior a confiabilidade que se pode ter na estimativa gerada.
Observa-se que com o modelo exponencial (Tabela 4 e Figura 8A) obteve o maior diferença entre o efeito pepita e o patamar $(0,98)$. As estimativas dos parâmetros do semivariograma exponencial foram: efeito pepita $=0,89$, patamar $=1,87 \mathrm{e}$ alcance $=242$ metros. Distância superior ao alcance apresenta distribuição aleatória e, portanto não são correlacionadas (VIEIRA, 2000).

Considerando-se os dados da Tabela 4, pode-se verificar que a dependência espacial da CTC Total foi moderada e bem definida. 

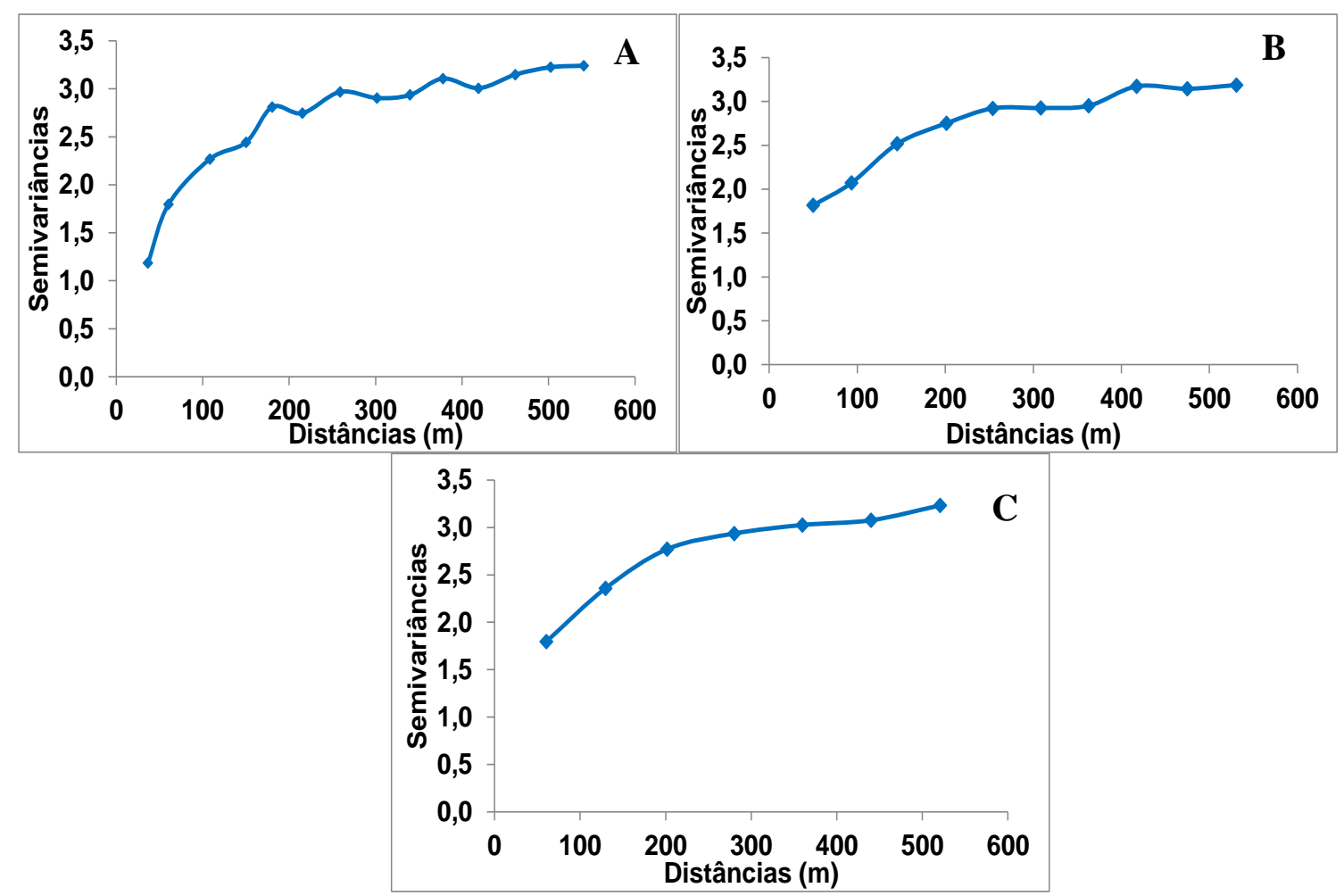

Figura 7. Semivariâncias com passo de 40 (A), 56 (B) e 80 metros (C) para CTC Total $\left(\mathrm{cmol}_{\mathrm{c}} \mathrm{dm}^{-3}\right)$, na camada $0-20 \mathrm{~cm}$ do solo, sem retirada de tendência.

Tabela 3. Análise de regressão para a retirada de tendência linear (L) e quadrática (Q) para CTC Total $\left(\mathrm{cmol}_{\mathrm{c}} \mathrm{dm}^{-3}\right), 0-20 \mathrm{~cm}$ do solo.

\begin{tabular}{ccccc}
\hline Fonte de & $\begin{array}{c}\text { Graus de } \\
\text { Liberdade }\end{array}$ & $\begin{array}{c}\text { Soma dos } \\
\text { Quadrados }\end{array}$ & $\begin{array}{c}\text { Quadrado } \\
\text { Médio }\end{array}$ & Teste F \\
\hline Regressão-L & 2 & 73,77 & 337,9 & 118,5 \\
Desvio-L & 237 & 675,9 & 2,852 & \\
Total-L & 239 & 749,7 & & 46,8 \\
\hline Regressão-Q & 5 & 115,9 & 126,7 & \\
Desvio-Q & 234 & 633,7 & 2,708 & \\
Total-Q & 239 & 749,7 & & \\
\hline
\end{tabular}

Coeficiente de Determinação do Ajuste: $\mathrm{L}=9,84 \%$; $\mathrm{Q}=15,46 \%$

Coeficiente de Correlação: L=31,37\%; Q=39,32\%

Se considerarmos a variância espacial mínima como critério de seleção, o modelo exponencial é o que melhor se ajustou aos dados de CTC Total, pois tem-se assim o menor valor da variância não explicada ou não detectada, menor Razão de Aleatoriedade:

$$
R A=\frac{\text { Efeito Pepita }}{\text { Efeito Pepita+Patamar }} * 100,(\text { Eq. 2) }
$$

e o maior IDE (Tabela 4). Utilizando-se o modelo exponencial, verificou-se que a distância máxima de dependência espacial, ou seja, a distância máxima em que os dados da CTC Total são correlacionados entre si é de 242 metros, então, a amostragem de solo pode ser realizada em uma grade amostral com esta premissa.

De acordo com Souza, Cogo e Vieira (1997), no estudo da continuidade espacial de nutrientes do solo o objetivo principal do uso dos semivariogramas é a determinação do número ideal de amostras. De acordo com esses autores, conhecendo-se o alcance da dependência espacial, define-se o raio de amostragem e garante-se uma amostragem 
futura nas mesmas condições. Cherubin et al. coeficiente linear $=0 ;$ coeficiente angular (2014) concluem que o estudo da (b)=1; coeficiente decorrelação $(r)=1$; média variabilidade espacial é eficiente para dos erros=0; variância=1; Índice Jack-Knifing definição de grade amostral futura.

$(\mathrm{IJK})=0$, considerando-se valores anteriores;

Para validação do modelo utilizou-se e Raiz Quadrada do Erro Médio

o Jack-Knifing, cujos valores ideais são: (RMSE)=valor mínimo.

Tabela 4. Estimativas dos parâmetros dos semivariogramas: exponencial, gaussiano e esférico com passo de 56 metros, Índice de Dependência Espacial (IDE) e Razão de Aleatoriedade (RA).

\begin{tabular}{ccccccccc}
\hline Modelo & $\begin{array}{c}\text { Efeito } \\
\text { Pepita } \\
\text { (EP) }\end{array}$ & $\begin{array}{c}\text { Patamar } \\
(\mathrm{P})\end{array}$ & EP + P & Alcance & IDE & RA & $\mathrm{R}^{2}$ & Dependência \\
\hline Exponencial & 0,89 & 1,87 & 2,76 & 242 & $68 \%$ & $32 \%$ & $96 \%$ & Moderada \\
Gaussiano & 1,61 & 1,13 & 2,74 & 213 & $41 \%$ & $59 \%$ & $98 \%$ & Moderada \\
Esférico & 1,36 & 1,37 & 2,73 & 244 & $50 \%$ & $50 \%$ & $98 \%$ & Moderada \\
\hline
\end{tabular}

Pode-se observar nas Figuras 8 e 9 que em relação ao coeficiente linear, os três modelos ficaram próximos do ideal (valor zero), com o modelo exponencial diferindo dos outros por volta de 0,005 próximos de dez vizinhos, enquanto para o coeficiente angular até por volta de quinze vizinhos temse uma proximidade muito grande entre os três modelos.

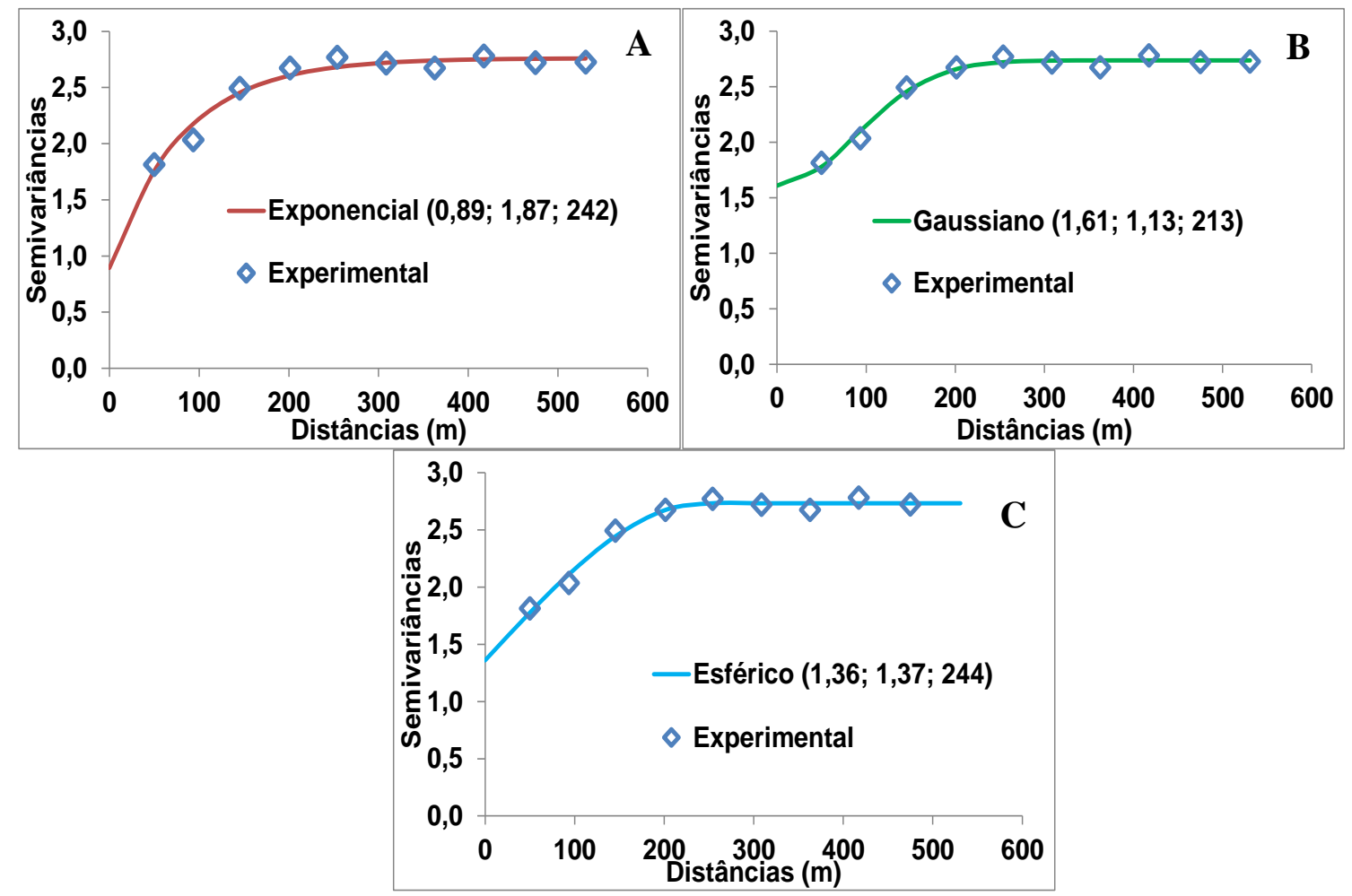

Figura 8. Semivariograma experimental ajustado ao Modelo Exponencial (A), Gaussiano (B) e Esférico (C) para CTC Total $\left(\mathrm{cmol}_{\mathrm{c}} \mathrm{dm}^{-3}\right)$, com step de 56 metros.

O coeficiente de correlação coloca os três modelos muito próximos, de modo que 
com dez vizinhos eles estão praticamente juntos. Em relação à média dos erros, os que 0,005 . Com relação às variâncias, os três modelos se aproximam do valor ideal (1), com diferenças entre si de 0,02 na região com modelos com aproximadamente 10 vizinhos apresentam diferença entre si menores 10 vizinhos, com leve vantagem para osmodelos esférico e gaussiano.

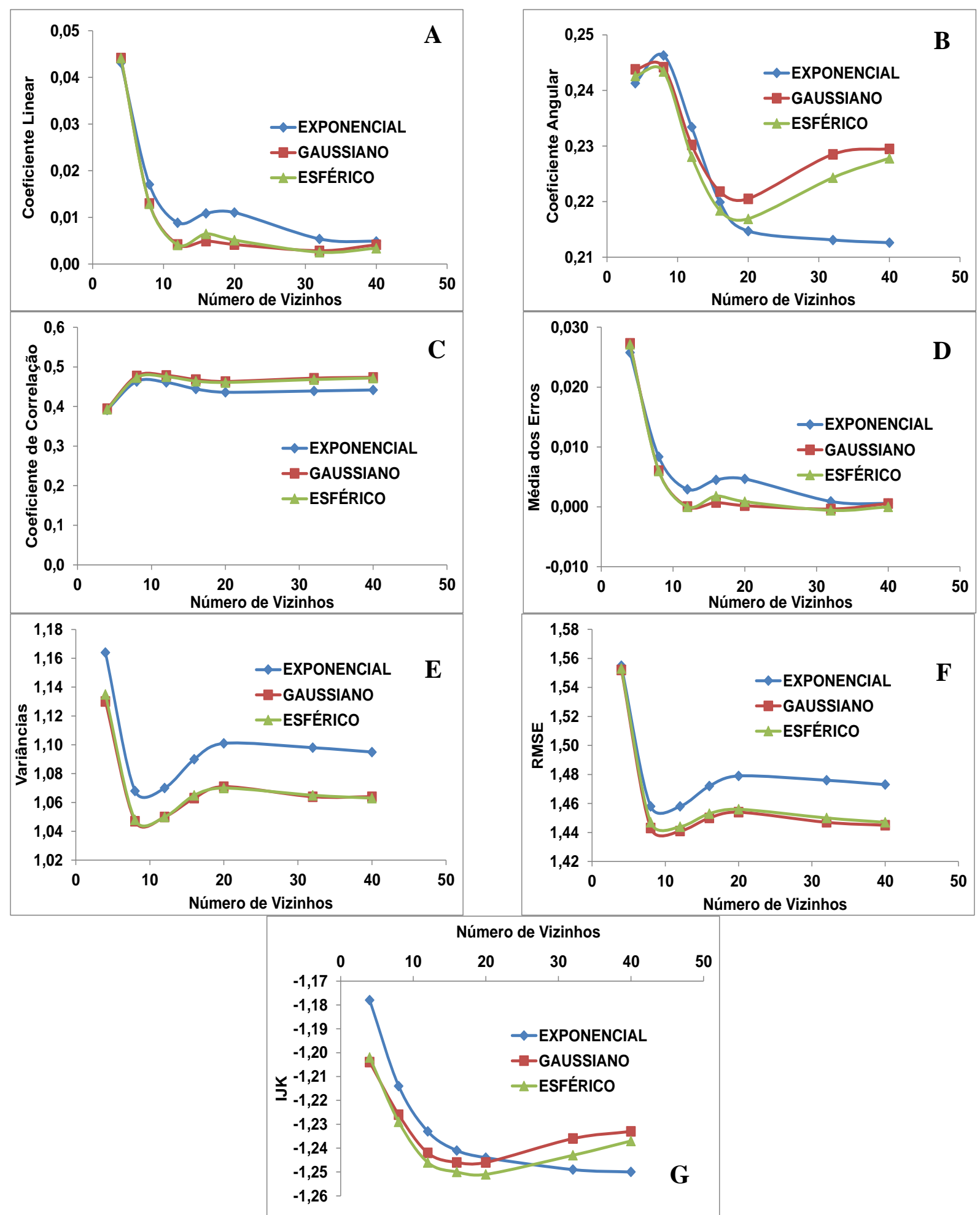

Figura 9. Gráficos com a validação para os Modelos estudados, com as variáveis: Correlação Linear (A), Correlação Angular (B), Coeficiente de Correlação (C), Média dos Erros (D), Variâncias (E), RMSE (F) e Índice Jack-Knifing-IJK (G). 
Tendo-se encontrado dependência possíveis zonas de manejo diferenciado para espacial moderada da CTC Total, faz-se o solo estudado. necessário uso de técnicas como a krigagem (Figura 10), para que se possa delimitar
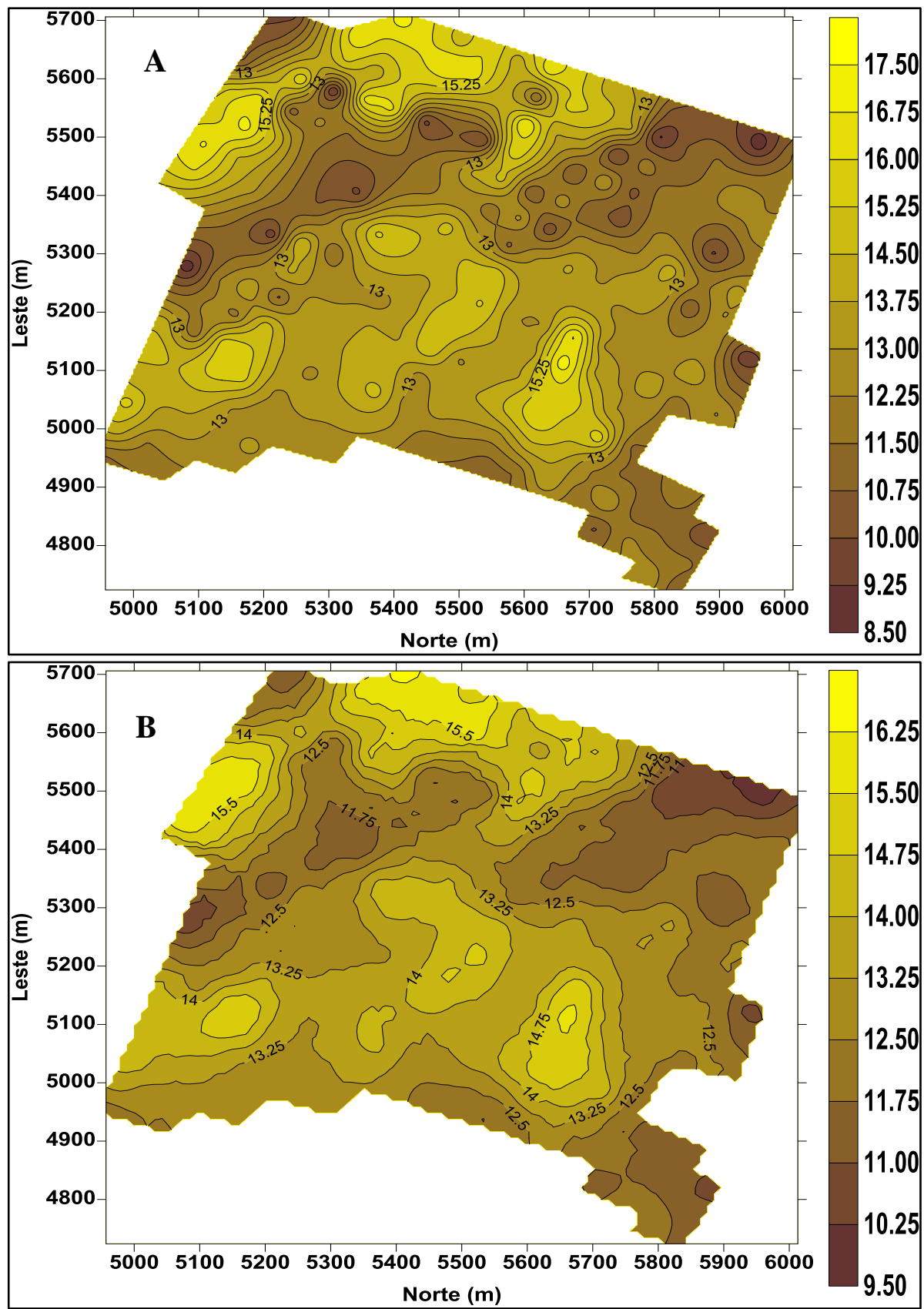

Figura 10. Mapa de isolinhas da área amostral, antes (A) e após a krigagem (B), considerando-se semivariograma ajustado ao modelo exponencial e usando-se sete vizinhos para CTC Total $\left(\mathrm{cmol}_{\mathrm{c}} \mathrm{dm}^{-3}\right)$, na camada de $0-20 \mathrm{~cm}$ do solo.

O índice Jack-Knifing coloca o próximas etapas, visto que os parâmetros modelo exponencial em vantagem com anteriores não negam esta afirmação. Por fim, número de vizinhos menores que 20, o qual a RMSE mostra uma leve vantagem para os representa um bom parâmetro para a modelos esférico e gaussiano em relação ao definição do modelo a ser usado nas exponencial, porém na região com dez 
vizinhos a diferença aproxima-se de 0,02. Com a realização da validação e mantendo-se os critérios avaliados nas etapas anteriores, pode-se reafirmar a escolha do modelo exponencial, confirmado pela avaliação do índice Jack-Knifing.

Sendo a krigagem uma técnica que permite estimar valores de variáveis para locais onde as mesmas não foram medidas ou foram perdidas, a partir de valores adjacentes interdependentes, foi realizada a krigagem ordinária, levando-se em consideração sete vizinhos para cada ponto calculado, com os parâmetros do semivariograma exponencial. O mapa de isolinhas da área amostral mostrou-se eficiente para a avaliação da distribuição da CTC Total, concordando com Neves Neto et al. (2013) que também utilizaram este tipo de mapa.

Nota-se que, após a krigagem, houve uma suavização das camadas da CTC Total (Figura 10B). Os mapas da área amostral mostram que existe uma concentração dos menores valores de CTC Total na região, onde está o talvegue, correspondente à região mais funda de um vale, no qual passam as águas de chuva, rios e lagoas delineando a direção natural deste curso, sendo sua linha a parte mais rápida da correnteza, lixiviando-se os nutrientes do solo. Existe uma indicação de que o alagamento provocado pelas lagoas presentes nesta fazenda, promoveu uma perda de nutrientes na sua área de influência. Podese inferir que a lixiviação através do alagamento e a posterior erosão no momento da saída da água, com o carreamento de solo rico em nutrientes desta região, promoveram esta aglutinação, Rosa Neto (2009) e Shing e Oliveira (2011), analisando argila para o mesmo banco de dados obtiveram comportamento similar. Este comportamento também foi encontrado nos trabalhos realizados por Oliveira (2003) para Cálcio, Santana (2008) e Santos e Oliveira (2011) para Silte.

\section{CONCLUSÕES}

A análise exploratória foi eficiente para detectar valores discrepantes (outliers).
Foi possível identificar dependência espacial nos dados da CTC Total $\left(\mathrm{cmol}_{\mathrm{c}} \mathrm{dm}^{-}\right.$ 3) na camada do solo a $0-20 \mathrm{~cm}$.

O modelo que melhor ajusta aos dados foi o exponencial.

A aplicação do método geoestatístico, em trabalhos futuros possibilitará a redução do número de amostras de solo coletadas para avaliação da necessidade da aplicação de nutrientes.

\section{AGRADECIMENTOS}

À equipe da Embrapa Solos pelo fornecimento dos dados, especialmente aos pesquisadores Dr. Enio Fraga da Silva, Dr. José Ronaldo de Macedo, Dra. Helga Hissa Manzatto e Dr. Cláudio Luiz Capeche.

Este artigo foi aprovado para publicação pelo Comitê de Publicações da Embrapa Soja sob o número 32/2013.

\section{REFERÊNCIAS}

BOLFE, E. L.; GOMES, J. B. V.; FONTES, H. R. Variabilidade espacial de atributos do solo como subsídio para produção integrada de frutas em perímetro irrigado no nordeste do Brasil. In: XIII Simpósio Brasileiro de Sensoriamento Remoto, Florianópolis, SC, INPE. Anais... Florianópolis, SC, 2007. p. 67-74.

CAMBARDELLA, C. A.; MOORMAN, T. B.; NOVAK, J. M.; PARKIN, T. B.; KARLEN, D. L.; TURCO, R. F.; KONOPKA, A. E. Field-scale variability of soil properties in Central Iowa Soils. Soils Sci. Am. J., v. 58, p. 1501-1511, 1994.

CARVALHO, J. R. P. de, VIEIRA, S.R. Avaliação e comparação de estimadores de krigagem para variáveis agronômicas Uma proposta. Série Documentos. ISSN 1677-9274. 2001.

CARVALHO, J. R. P. de, SILVEIRA, P. M. da. VIEIRA, S.R. Geoestatística na determinação da variabilidade espacial de características químicas do solo sob diferentes preparos. Pesquisa Agropecuária 
Brasileira, PAB. Brasília, DF, v.37, n.8, p.1151-1159, 2002.

CARVALHO, L. A. de; MEURES, I.; SILVA JUNIOR, C. A. da; CAVALIERI, K. M. V.; SANTOS, C. F. B. Dependência espacial dos atributos físicos de três classes de solos cultivados com cana-de-açúcar sob colheita mecanizada. Revista Brasileira de Engenharia Agrícola e Ambiental, v.15, n.9, p.940-949, Campina Grande, PB. 2011.

CAVAlCANTE, E. G. S., ALVES, M. C.; SOUZA, Z. M. de; PEREIRA, G. T. Variabilidade espacial de atributos físicos do solo sob diferentes usos e manejos. Revista Brasileira de Engenharia Agrícola e Ambiental, v.15, v.3, p. 237-243, Campina Grande, PB. 2011.

CHERUBIN, M. R.; SANTI, A. L.; EITELWEIN, M. T.; MENEGOL, D. R.; DA ROS, C. O.; PIAS, O. H. de C.; BERGHETTI, J. Eficiência de malhas amostrais utilizadas na caracterização da variabilidade espacial de fósforo e potássio. Ciência Rural, Santa Maria, v.44, n.3, p.425432. 2014.

EMBRAPA. Manual de métodos de análises de solo. Centro Nacional de Pesquisa de Solos. 2. ed. rev. Atual. Rio de Janeiro, RJ, (EMBRAPA-CNPS. Documentos: 1). 1997. 212p.

EMBRAPA. Levantamento detalhado dos solos da Fazenda Angra-PESAGRO-Rio. Embrapa Solos. Rio de Janeiro, RJ, (Embrapa Solos. Boletim de Pesquisa e Desenvolvimento: 38), 2003. 93p.

GREGO, C. R. e VIEIRA, S. R. Variabilidade espacial de propriedades físicas do solo em uma parcela experimental. Revista Brasileira de Ciência do Solo. v.29. p. 169-177. 2005.

HOAGLIN, D.C., MOSTELLER, F., TUKEY, J.W. Análise exploratória de dados. Técnicas robustas. Edições Salamandra. Lisboa. 446p. 1992.

KOPPE, V. C.; COSTA, J. F. C. L.; KOPPE, J. C. Coordenadas cartesianas $\mathrm{x}$ coordenadas geológicas em geoestatística: aplicação à variável vagarosidade obtida por perfilagem acústica. Revista Escola de Minas. v. 59. p. 25-30. 2006.

NEVES NETO, D. N.; SANTOS, A. C. dos; SANTOS, P. M.; MELO, J. C.; SANTOS, J. S. Análise espacial de atributos do solo e cobertura vegetal em diferentes condições de pastagem. Revista Brasileira de Engenharia Agrícola e Ambiental, v.17, n.9, p.9951004, Campina Grande, PB. 2013.

OLIVEIRA, M. C. N. de. Métodos de estimação de parâmetros em modelos geoestatísticos com diferentes estruturas de covariâncias: uma aplicação ao teor de cálcio no solo. Piracicaba, SP. Tese de Doutorado - Escola Superior de Agricultura "Luiz de Queiroz", Universidade de São Paulo. 2003.140p.

OLIVEIRA, P. C. G.; FARIAS, P. R.S.; LIMA, H. V.; FERNANDES, A. R.; OLIVEIRA, F. A.; PITA, J. D. Variabilidade espacial de propriedades químicas do solo e da produtividade de citros na Amazônia Oriental. Revista Brasileira de Engenharia Agrícola e Ambiental, v.13, n.6, p.708-715, Campina Grande, PB. 2009.

ROSA NETO, E. A. Uso de Geoestatística no estudo espacial de dados e propriedades físicas em solos hidromórficos. Monografia - Universidade Estadual de Londrina Londrina, PR. 2009. 30p.

SANTANA, T. F. Aplicação de Método Geoestatístico a Dados de Pesquisa Utilizando o Software geoEas e variowin. Monografia - Universidade Estadual de Londrina - Londrina, PR. 2008. 49p.

SANTOS, E. O. de; GONTIJO, I., NICOLE, L. R. Variabilidade espacial de cálcio, 
magnésio, fósforo, potássio no solo e produtividade da pimenta-do-reino. Revista Brasileira de Engenharia Agrícola e Ambiental, v.16, n.10, p. 1062-1068, Campina Grande, PB, 2012.

SANTOS, M. R. G.; OLIVEIRA, M. C. N. de. Aplicação da geoestatística a dados de teor de silte em solo hidromórfico. Resumos expandidos da VI Jornada Acadêmica da Embrapa Soja, 6. 2011, Londrina, PR. Embrapa Soja. p.68-71. 2011.

SHING, L. R.; OLIVEIRA, M. C. N. de. Uso da geoestatística na determinação da variabilidade espacial do teor de argila no solo. Resumos expandidos da VI Jornada Acadêmica da Embrapa Soja, 6. 2011, Londrina, PR. Embrapa Soja. p.79-82. 2011.

SILVA JUNIOR, J. F. da; PEREIRA, G. T.; CAMARGO, L. A.; MARQUES JÚNIOR, J. Métodos geoestatísticos na modelagem espacial do diâmetro médio do cristal da goethita. Revista Brasileira de Engenharia Agrícola e Ambiental, v.17, n.11, p.11271134, Campina Grande, PB. 2013.

SOUZA, L. S.; COGO, N. P.; VIEIRA, S. R. Variabilidade de propriedades físicas e químicas do solo em pomar cítrico. Revista Brasileira de Ciência do Solo. Campinas, SP, v.21, n.3, p.1-10, 1997.

SOUZA, Z. M. de; CERRI, D. G. P.; COLET, M. J.; RODRIGUES, L. H. A.; MAGALHÃES, P. S. G.; MANDONI, R. J. A. Análise dos atributos do solo e da produtividade da cultura da cana-de-açúcar com o uso da geoestatística e árvore de decisão. Ciência Rural, Santa Maria, v.40, p.840-847, 2010a.

SOUZA, Z. M.; MARQUES JÚNIOR, J.; PEREIRA, G. T. Geoestatística e atributos do solo em áreas cultivadas com cana-de-açúcar. Ciência Rural, Santa Maria. Online, v.40, n.1, 2010b.
SOUZA, Z. M.; SOUZA, G. S. de; MARQUES JÚNIOR, J.; PEREIRA, G. T. Número de amostras na análise geoestatística e na krigagem de mapas de atributos do solo. Ciência Rural, Santa Maria, v.44, n.2, p.261268, 2014.

VIEIRA, S. R. Geoestatística em estudos de variabilidade espacial do solo. In: Tópicos em Ciência do Solo. Viçosa: SBCS, 2000.

VIEIRA, S. R.; CARVALHO, J. R. P. de; GONZÁLES, A. P. Jack Knifing for Semivariogram Validation. Bragantia, v.69, p. 97-105. Suplemento. Campinas, SP, 2010.

VIEIRA, S. R.; MILLETE, J.; TOPP, G. C.; REYNOLDS, W. D. Handbook for geostatistical analysis of variability in soil and climate data. In: Tópicos em Ciência do Solo, 2. p. 1-45, Viçosa, MG. 2002.

VIEIRA, S. R.; NIELSEN, D. R.; BIGGAR, J. W. Spatial variability of field-mensured infiltration rate. Soil of American Journal, v.45, n.3, p.1040-1048. 1981.

VIEIRA, S.R.; HATFIELD, J.L.; NIELSEN, D.R.; BIGGAR, J.W. Geostatistical theory and application to variability of some agronomical properties. Hilgardia, v.51, n.3, p.1-75, 1983. 